\title{
Biochanin A induces anticancer effects in SK-Mel-28 human malignant melanoma cells via induction of apoptosis, inhibition of cell invasion and modulation of NF-кB and MAPK signaling pathways
}

\author{
PENG XIAO $^{1}$, BOWEN ZHENG $^{2}$, JIAMING SUN ${ }^{1}$ and JIA YANG $^{3}$ \\ ${ }^{1}$ Department of Plastic Surgery, Wuhan Union Hospital, Tongji Medical College, \\ Huazhong University of Science and Technology, Wuhan, Hubei 430030; ${ }^{2}$ Department of Dermatology, \\ The Second People's Hospital of Yunnan Province, Kunming, Yunnan 650021; ${ }^{3}$ Institute of Dermatology \\ and Venereology of Yunnan Province, Kunming University, Kunming, Yunnan 650214, P.R. China
}

Received December 30, 2016; Accepted June 22, 2017

DOI: $10.3892 / 01.2017 .6945$

\begin{abstract}
The present study aimed to investigate the antitumor activity of Biochanin A in SK-Mel-28 human malignant melanoma cells. An MTT assay was used to study the cytotoxic effects of Biochanin A. In vitro wound healing and invasion assays were used to investigate the effects on cell migration and invasion. Fluorescence microscopy using acridine orange/propidium iodide was used to study effects on cell morphology and apoptosis. Nuclear factor $(\mathrm{NF})-\kappa \mathrm{B}$ and mitogen-activated protein kinase (MAPK) protein expression levels were determined by western blot analysis. The results indicated that Biochanin A significantly inhibited the growth of SK-Mel-28 cells in a dose and time dependent manner. Treatment of the cells with Biochanin A induced apoptosis in a dose dependent manner. Additionally, Biochanin A led to inhibition of cell migration and invasion in a dose-dependent manner and upregulated the expression of key proteins in the $\mathrm{NF}-\kappa \mathrm{B}$ and MAPK signaling pathways.

\section{Introduction}

Malignant melanoma accounts for approximately $75 \%$ of the skin cancer-related deaths in the world. Malignant melanoma is an aggressive form of cancer and and its incidence has increased significantly in the recent past (1). Transformation of melanoma into the metastatic stage is a very complex process involving various biochemical processes like cell

Correspondence to: Dr Jia Yang, Institute of Dermatology and Venereology of Yunnan Province, Kunming University, 2 PuXin Road, Kunming, Yunnan 650214, P.R. China

E-mail: jiayang46@hotmail.com
\end{abstract}

Key words: malignant melanoma, Biochanin A, apoptosis, cytotoxicity, propidium iodide cycle disruption, evasion of apoptosis, abnormalities in the adhesion and dilapidation of the extracellular matrix, cell migration and cell invasion (1-3). As for treatment of malignant melanoma is concerned, there are only few chemotherapeutic agents available and those too have serious side effects (4). Patients with metastatic melanoma and who are not suitable for surgical resection, 5-(3,3-dimethyl-1-triazenyl)-1H-imidazole-4-carboxamide (DTIC) has been reported to be very effective chemotherapeutic agent (4). However, in single-dose regimen, its overall response rate is very poor and the duration of response is very low. Further, it has been reported that combination of DTIC with other chemotherapeutic agents like cisplatin, carmustine, tamoxifen etc has led to higher response rates $(4,5)$. But, unfortunately, this has also resulted in increased unwanted side-effects affecting the quality of life (5). In brief, the current treatment options for malignant melanoma especially metastatic melanoma are very limited and unsatisfactory. As a result there is a pressing need for development of new and effective chemotherapeutic agents for the treatment of malignant melanomas. Scientists are exploring the traditional and seemingly safe natural medicines for the anticancer therapeutics. Plant based drugs including taxol, vincristine, vinblastine, topotecan, irinotecan, camptothecin, podophyllotoxin etc have been successfully used for the treatment of a variety of human tumors. These compounds have been used as prototypes for the design and development of future anticancer drugs (6). Since, most of these drugs are associated with lot of side effects, there is need to explore less toxic and more effective drugs. Consistent with this, isoflavones are generally well tolerated and have not exhibited toxicity in humans (7) and may therefore prove potential candidates for anticancer drug development. Biochanin A is one such flavonoid and has been reported to exhibit anticancer activity against a range of cancer cells which include prostate cancer (8), pancreatic cancer (9) and breast cancer (7). However, the anticancer activity of Biochanin A has not been evaluated against human malignant melanoma cells. Therefore the aim of the present investigation was to evaluate the 
anticancer and apoptotic effects of Biochanin A in SK-Mel-28 human malignant melanoma cells along with investigating its effects on cell migration and invasion, cell cycle arrest and nuclear factor (NF)- $\mathrm{B}$ and mitogen-activated protein kinase (MAPK) signalling pathway.

\section{Materials and methods}

Chemical and other biochemical reagents. Biochanin A (95\% purity by HPLC), 3-(4,5-dimethylthiazol-2-yl)-2,5-diphenyltetrazolium bromide (MTT), DMSO, Penicillin, streptomycin were purchased from Sigma-Aldrich (St. Louis, MO, USA). Fetal bovine serum (5\% heat-inactivated), $3.5 \%$ trypsin, Dulbecco's modified Eagle's medium were purchased from Gibco (Carlsbad, CA, United States). Acridine orange (AO) and propidium iodide (PI) were purchased from Beyotime Institute of Biotechnology (Shanghai, China).

Cell line and cell culture conditions. Human malignant melanoma cell line (SK-Mel-28) was purchased from the Cell Institute, Chinese Academy of Sciences (Shanghai, China). The cells were cultured in Dulbecco's modified Eagle's medium comprising of 5\% heat-inactivated fetal bovine serum, $100 \mathrm{U} / \mathrm{ml}$ penicillin and $100 \mu \mathrm{g} / \mathrm{ml}$ streptomycin with $5 \% \mathrm{CO}_{2}$ at $37^{\circ} \mathrm{C}$.

Cell cytotoxicity assay. In brief, human malignant melanoma cell line (SK-Mel-28) cells at a density of $2 \times 10^{6}$ cells $/ \mathrm{ml}$ were cultured in a 96 -well chamber slide for $24 \mathrm{~h}$ before using them in the experiment. SK-Mel-28 cells were then treated with $0,10,25,50,75$ and $100 \mu \mathrm{M}$ dose of Biochanin A dissolved in DMSO (0.1\%) for 48 and 72 h. Subsequently, MTT was added into each well and then again cultured for $3 \mathrm{~h}$ before the supernatant was discarded. The formazan crystals formed were dissolved in DMSO and the absorbance values were examined on Automated Microplated Reader (BioTek Instruments, Inc., Winooski, VT, USA) at $570 \mathrm{~nm}$. Cytotoxicity was represented as the concentration of Biochanin A inhibiting cell growth by $50 \%$ ( $\mathrm{IC}_{50}$ value).

In vitro wound healing assay. Human malignant melanoma cell line (SK-Mel-28) $\left(1 \times 10^{6}\right.$ cells $\left./ \mathrm{ml}\right)$ were seeded in a 6-well plate and incubated at $37^{\circ} \mathrm{C}$ till $100 \%$ confluent monolayer of cells was attained. A straight cell-free wound was made using a $100 \mathrm{ml}$ pipette tip. To each well, different doses $(0,10,50$ and $100 \mu \mathrm{M}$ ) of Biochanin A were added and the cells were washed with PBS to eliminate any cellular debris. After that the cells were stained using $0.5 \%$ crystal violet powder for $15 \mathrm{~min}$. The arbitrarily selected fields were chosen and photographed using an inverted light microscope (IX71; Olympus, Tokyo, Japan). The percentage of cells that migrated into the scratched area were calculated and length of wounds was determined by Image J (version 1.46) software.

Invasion assay. After the SK-Mel-28 cells were washed with PBS, the cells were again suspended in serum free medium. Then $400 \mu$ l of cell suspension $\left(2 \times 10^{6}\right.$ cells $\left./ \mathrm{ml}\right)$ was added to the upper chamber coated with Matrigel membrane (Millipore, Billerica, MA, USA) while as lower chamber was filled with medium containing $5 \%$ FBS. SK-Mel-28 cells were incubated with various doses $(0,10,50$ and $100 \mu \mathrm{M})$ of Biochanin A for

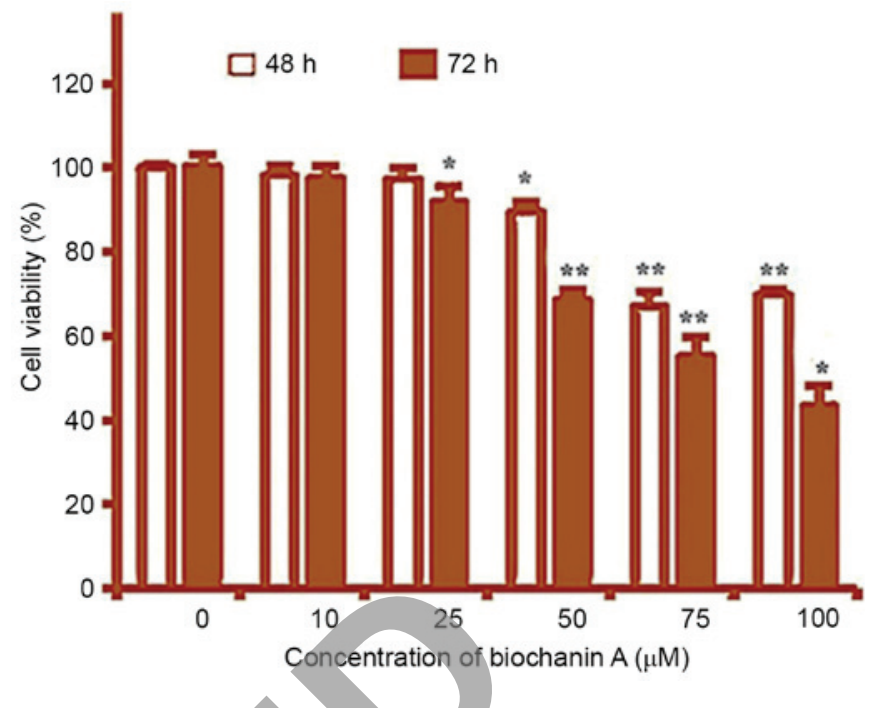

Figure 1. Biochanin A induces concentration-dependent cytotoxicity in human malignant melanoma cells (SK-Mel-28). The cells were treated with $0,10,25,50,75$ and $100 \mu \mathrm{M}$ dose of Biochanin A and then incubated for 48 and 72 h. Cell cytotoxicity was assessed by MTT assay. Data are shown as the mean \pm standard deviation of three independent experiments. ${ }^{*} \mathrm{P}<0.05$, ${ }^{* *} \mathrm{P}<0.01$ vs. $0 \mu \mathrm{M}$ (control).

$1 \mathrm{~h}$ at $25^{\circ} \mathrm{C}$. After $48 \mathrm{~h}$, the cells which had still remained in the upper face of the filters were removed, and the cells which had travelled to the lower face of the filters were fixed with $90 \%$ methanol and then stained with $0.35 \%$ crystal violet and then counted using light microscope.

Fluorescence microscopy using AO/PI. Human malignant melanoma cells (SK-Mel-28) were plated on a chamber slide at a density of $2 \times 10^{6}$ cells per chamber and then treated with varying doses $(0,10,50$ and $100 \mu \mathrm{M})$ of Biochanin A and incubated for $48 \mathrm{~h}$ before $10 \mu \mathrm{g} / \mathrm{ml}$ of $\mathrm{AO}$ and $10 \mu \mathrm{g} / \mathrm{ml}$ of PI were added to each chamber. The images of the treated and untreated cells were captured by a UV fluorescence microscope (Olympus Optical Co., Ltd, Tokyo, Japan). The images were taken at x200 magnification so that the evidence of apoptosis in clear.

Western blot analysis. A western blot assay was carried out to examine the expression of proteins responsible for the anticancer effect. SK-Mel-28 cells were harvested and lysed with RIPA buffer and the collected protein samples were examined by bichinconinic acid protein assay kit for protein quantification. Approximately $200 \mu \mathrm{g}$ of cellular protein from each sample was applied to 8-10\% SDS-polyacrylamide gels and probed with specific antibodies followed by exposure to horseradish peroxidase-conjugated goat anti-mouse antibodies. Blots were then developed using the West Pico Chemiluminescent substrate (Pierce, Woburn, MA, USA). The antibodies against $\beta$-actin, p-MEK, MEK, NF- $\kappa B$ were obtained from Santa Cruz Biotechnology, Inc. (Santa Cruz, CA, USA).

Statistical analysis. All data were expressed as the mean \pm standard deviation. The means of the different groups were compared using one-way of variance (ANOVA) followed by the Student's t-test. Each experiment was performed at least 


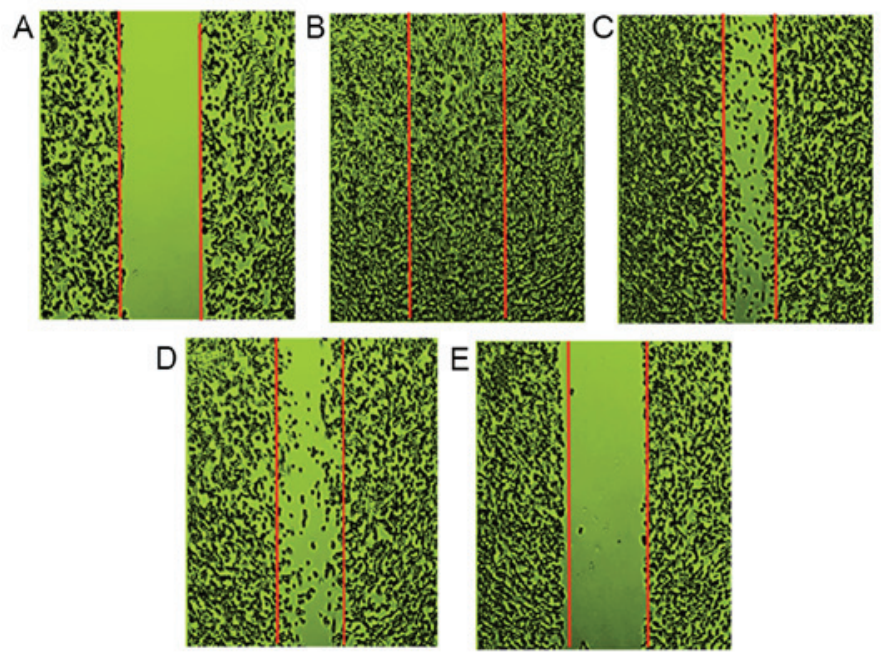

$\mathrm{F}$

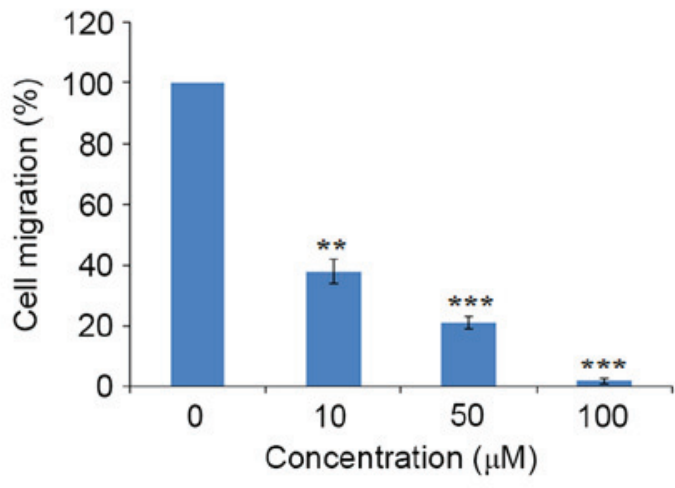

Figure 2. Biochanin A suppresses cell migration in SK-Mel-28 human malignant melanoma cells. In vitro wound healing assay was used to examine the effect of the drug on cell migration. Cells were treated with (B) 0 , (C) 10, (D) 50 and (E) $100 \mu \mathrm{M}$ dose of Biochanin A. (A) Shows an simulated wound prepared by scratching with a micropipette tip and the red lines represent the initial marks of the scratch. (F) Quantification of cell migration. Cell migration in untreated control was taken as $100 \%$. ${ }^{* *} \mathrm{P}<0.01$ and ${ }^{* * *} \mathrm{P}<0.001$ were considered statistically significant.
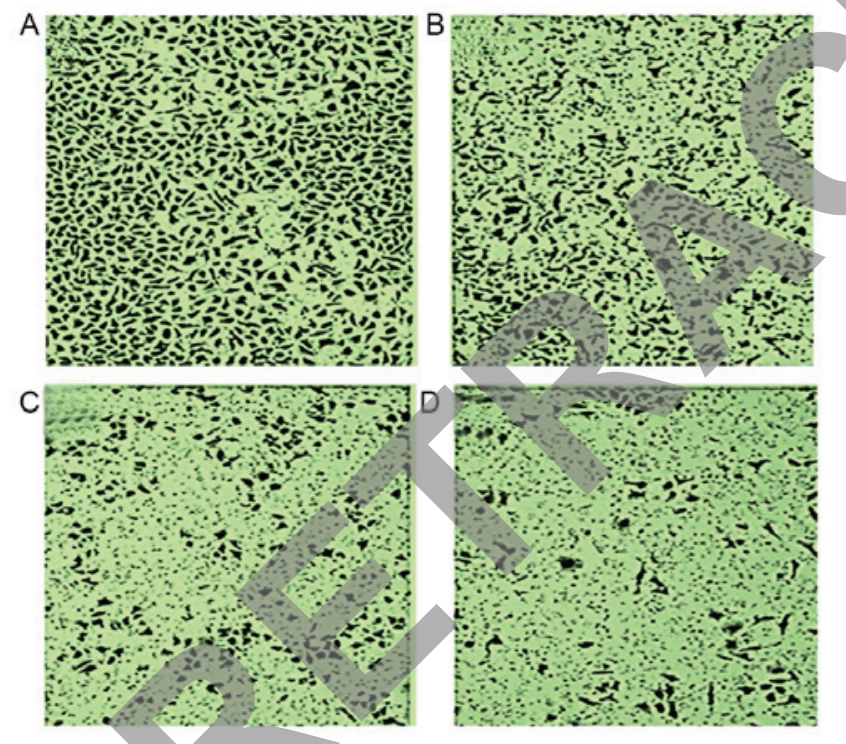

E

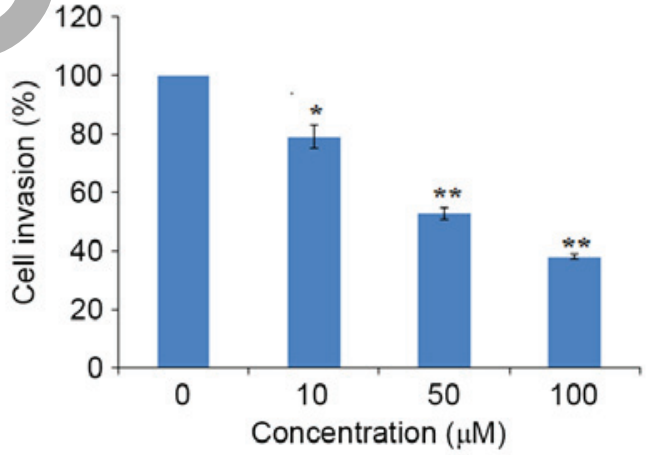

Figure 3. Inhibition of cell invasion in in SK-Mel-28 human malignant melanoma cells. The cells were treated with (A) 0 , (B) 10 , (C) 50 and (D) $100 \mu \mathrm{M}$ dose of Biochanin A for $48 \mathrm{~h}$ and the representative images were taken at x200 magnification. (E) Quantification of cell invasion. Cell invasion in untreated control was taken as $100 \%$. $\mathrm{P}<0.05$ and ${ }^{* *} \mathrm{P}<0.01$ were considered statistically significant.

three times. $\mathrm{P}<0.05$ was considered to indicate a statistically significant difference.

\section{Results}

Cytotoxic effects of Biochanin A in SK-Mel-28 human malignant melanoma cells. The cytotoxic effects of Biochanin A in SK-Mel-28 cells are depicted in Fig. 1. The cytotoxicity of the compound was evaluated by MTT assay using a range of concentrations including $0,10,25,50,75$ and $100 \mu \mathrm{M}$. The results point out that Biochanin $\mathrm{A}$ is a potent cytotoxic agent showing dose-dependent growth inhibition of these cancer cells. The cytotoxic effects of the compound were tested at 48 and $72 \mathrm{~h}$ incubation times and results revealed that the drug exerted time and concentration dependent anticancer effects.
In addition, it should be noted that the cytotoxic activity of Biochanin A was not significant at $10 \mu \mathrm{M}$ and the dose dependent only above $10 \mu \mathrm{M}$.

Biochanin A suppressed cell migration and invasion in SK-Mel-28 human malignant melanoma cells. The effect of Biochanin A on the cancer cell migration and invasion in SK-Mel-28 human malignant melanoma cells was evaluated by in vitro wound healing assay and invasion assay respectively. The results which are depicted in Fig. 2 revealed that a wound scratch in vehicle-treated control cells was virtually fully closed after $48 \mathrm{~h}$ of incubation. However, treatment with $0,10,50$ and $100 \mu \mathrm{M}$ dose of Biochanin A (Fig. 2B-E) led to suppression of cell migration in a dose-dependent manner. As can be seen from Fig. 3A-D, Biochanin A also inhibited cancer cell invasion 

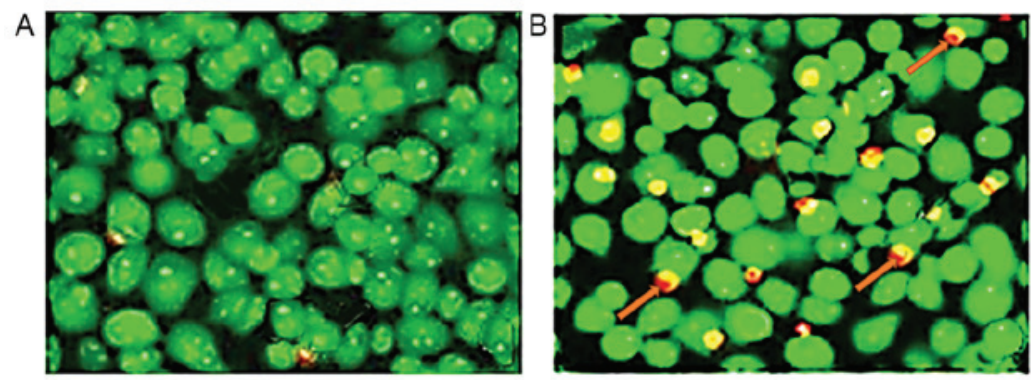

E
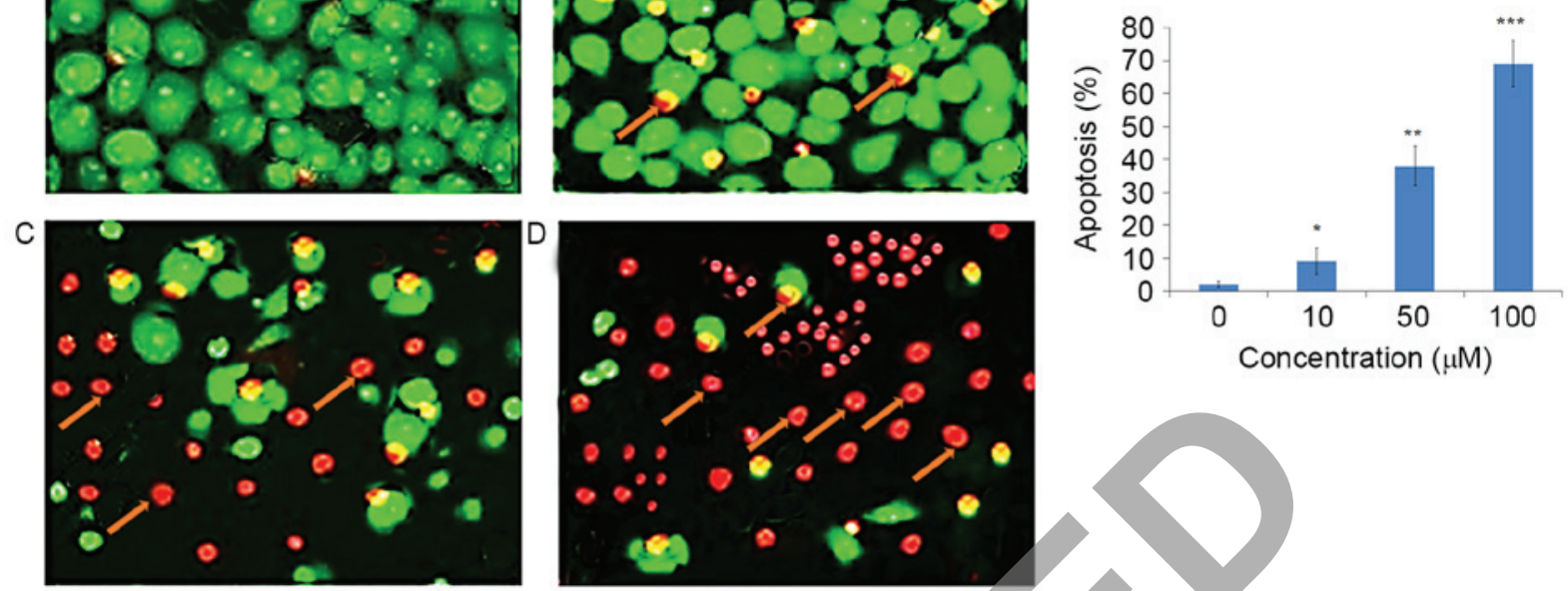

Figure 4. Morphological evaluation of SK-Mel-28 human malignant melanoma cells using acridine orange/propidiumiodide (AO/PI) staining in combination with fluorescence microscopy. Cells were treated with (A) 0 , (B) 10, (C) 50 and (D) $100 \mu \mathrm{M}$ of Biochanin A for $48 \mathrm{~h}$. Green fluorescence indicates normal cells while orange/red fluorescence indicates apoptotic cells. (E) Quantification of apoptotic cells ${ }^{*} \mathrm{P}<0.05,{ }^{* *} \mathrm{P}<0.01$ and ${ }^{* * *} \mathrm{P}<0.001$ were considered statistically significant.

in a dose-dependent manner. Thus these results indicate that Biochanin A might show anticancer effects by inhibiting cancer cell migration and invasion. Unlike MTT assay, the inhibition of cell migration and invasion were observed even at the lowest concentration of $10 \mu \mathrm{M}$ Chemotherapeutic agents which suppress cancer cell migration and invasion are believed to be promising antitumor drugs because cancer cell migration and invasion have a direct relationship with cancer metastases.

Biochanin A induces apoptosis in SK-Mel-28 human malignant melanoma cells. $\mathrm{AO} / \mathrm{PI}$ staining was also done to examine the apoptosis-inducing effects of Biochanin A in SK-Mel-28 human malignant melanoma cells. As compared to the untreated control cells which indicated homogenous green fluorescence, the Biochanin A-treated cells with 10,50 and $100 \mu \mathrm{M}$ dose showed orange and red fluorescence indicating onset of apoptosis in these cells (Fig. 4A-D). The population of these apoptotic cells (red fluorescence) increased with increasing doses of Biochanin A. Unlike the results of MTT assays, the results in apoptosis assay also showed apoptosis at $10 \mu \mathrm{M}$ of Biochanin A.

Biochanin A leads to upregulation of $N F-\kappa B$ and MAPK signalling pathways. Western blot assay was used to study the expression levels of various key proteins involved in the anticancer action of Biochanin A in SK-Mel-28 human malignant melanoma cells. Biochanin A treatment to SK-Mel-28 cells led to activation of NF- $\mathrm{KB}$ in a dose-dependent manner (Fig. 5). Biochanin A also resulted in activation (upregulation) of the MAPK signalling pathways. Biochanin A significantly increased the phosphorylation of MAPK proteins. The upregulation of MAPK was seen to follow dose dependent pattern.

\section{Discussion}

Malignant melanoma accounts for about $75 \%$ of the skin cancer-related deaths in the world and there are limited

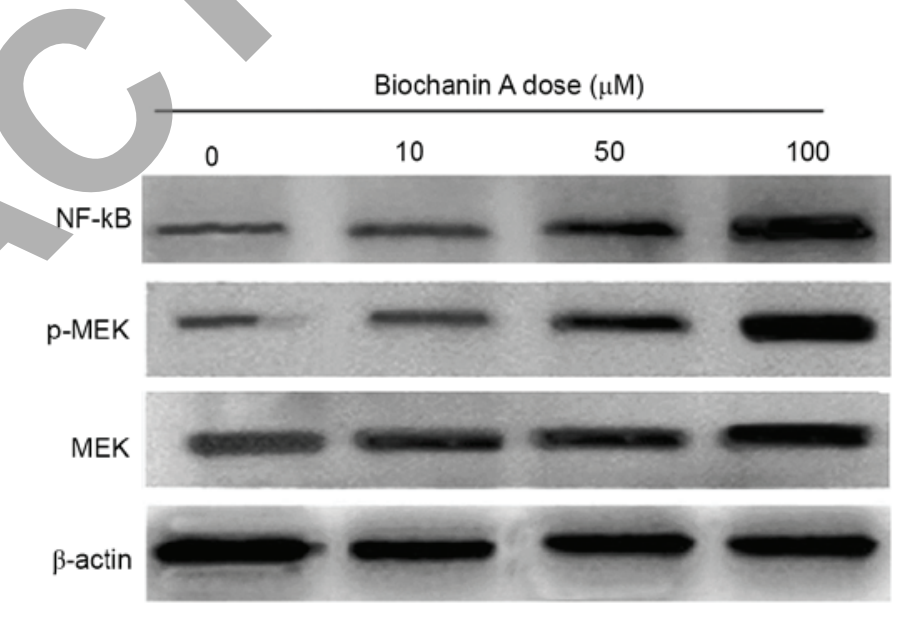

Figure 5. Effect of Biochanin A on the activation of NF- $\kappa \mathrm{B}$, mitogen-activated protein kinase signalling pathway in SK-Mel-28 human malignant melanoma cells. The cells were treated with $0,10,50$ and $100 \mu \mathrm{M}$ dose of Biochanin A for $48 \mathrm{~h}$ and then specific antibodies were used to determine the expression

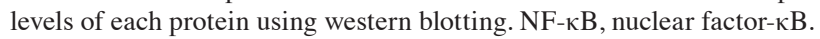

treatment options available for its treatment. While as a number of drugs have been isolated from plants, many of them exibit toxicity. However, isoflavonoids have been reported to exhibit little or no cytotoxicity in human (7) and therefore may prove important targets for development of cancer chemotherapy. Biochanin A is a one such plant flavonoid and is mostly found in red clover, soy, peanuts and chickpea (10). Flavonoids are one of the common constituents in the human diet. Flavonoids have been reported to show chemopreventive action against different cancers. They are reported to show anticancer action by various mechanisms including cell cycle arrest, induction of apoptosis, carcinogen inactivation, and suppression of angiogenesis, antioxidative capacity and reversal of multidrug resistance (11-16).

In the present study, our main objective was to evaluate the antitumor activity of Biochanin A in SK-Mel-28 human 
malignant melanoma cells. Its effects on apoptosis, cell migration and invasion were also examined along with studying its effects on $\mathrm{NF}-\kappa \mathrm{B}$ and MAPK signalling pathway. The results suggest that Biochanin A inhibits cancer cell growth in SK-Mel-28 melanoma cells in a concentration dependent manner as well as time-dependent manner. Biochanin A also led to onset of orange/red fluorescence in these cells and the intensity of this red fluorescence increased with increasing dose of the drug indicating that Biochanin A induces apoptosis in SK-Mel-28 melanoma cells. These preliminary results will form basis for future exploration of the apoptosis inducing mechanism of Biochanin A in presence of zVAD with appropriate controls and in different cell lines. Further results revealed that Biochanin A also led to the inhibition of cell migration and cell invasion in these cells. Biochanin A might display antitumor effects by impeding cancer cell migration and invasion. Chemotherapeutic agents which suppress cancer cell migration and invasion are believed to be promising antitumor drugs because cancer cell migration and invasion have a direct relationship with cancer metastases $(17,18)$. The migration and invasion of cancer cells are significant events in metastasis (17). Consequently, suppression of the invasion and metastasis of cancer cells has been thought to be a reasonable approach for the malignancy treatment (17). Western blot assay showed that Biochanin A led to upregulation of $\mathrm{NF}-\kappa \mathrm{B}$ and MAPK signalling pathways. While as biochanin did enhance the expression of NF- $\mathrm{NB}$ and MAPK, further investigations would confirm the involvement of these signalling pathways in biochanin induced anticancer activity. Moreover, the evaluation of the effect Biochanin A on MAPK downstream targets may aslo prove useful in and needs to be thoroughly studied in future. Taken together, the results indicate that Biochanin A may prove an important lead molecule.

In brief, the current study reports the antitumor and apoptotic effects of Biochanin A in SK-Mel-28 human malignant melanoma cells. The current study also reports that the anticancer effects of the compound were mediated via inhibition of cell migration and invasion Moreover the molecule also exerts its effects on NF- $\mathrm{BB}$ and MAPK signalling pathways.

\section{References}

1. Hussein MR, Haemel AK and Wood GS: Apoptosis and melanoma: Molecular mechanisms. J Pathol 199: 275-288, 2003.
2. Soengas MS and Lowe SW: Apoptosis and melanoma chemoresistance. Oncogene 22: 3138-3151, 2003.

3. Senderowicz AM: Novel small molecule cyclin-dependent kinases modulators in human clinical trials. Cancer Biol Ther 2 (4 Suppl 1): S84-S95, 2003.

4. Comis RL: DTIC (NSC-45388) in malignant melanoma: A perspective. Cancer Treat Rep 60: 165-176, 1976.

5. McClay EF and McClay ME: Tamoxifen: Is it useful in the treatment of patients with metastatic melanoma? J Clin Oncol 12: 617-626, 1994.

6. Cragg GM, Boyd MR, Cardellina JH, Grever MR, Schepartz SA, Snader KM and Matthew S: Role of Plants in the National cancer Institute drug discovery and development program. ACS Symposium Series 534: 80-95, 2009.

7. Sehdev V, Lai JC and Bhushan A: Biochanin A modulates cell viability, invasion, and growth promoting signaling pathways in HER-2-positive breast cancer cells. J Oncol 2009: 121458, 2009.

8. Seo YJ, Kim BS, Chun SY, Park YK, Kang KS and Kwon TG: Apoptotic effects of genistein, biochanin-A and apigenin on LNCaP and PC-3 cells by $\mathrm{p} 21$ through transcriptional inhibition of polo-like kinase-1. J Korean Med Sci 26: 1489-1494, 2011.

9. Bhardwaj V, Tadínada SM, Jain A, Sehdev V, Daniels CK, Lai JC and Bhushan A: Biochanin A reduces pancreatic cancer survival and progression. Anticancer Drugs 25: 296-302, 2014.

10. Medjakovic S and Jungbauer A: Red clover isoflavones Biochanin A and formononetin are potent ligands of the human aryl hydrocarbon receptor. J Steroid Biochem Mol Biol 108: 171-177, 2008.

1. Hodek P, Trefil P and Stiborová M: Flavonoids-Potent and versatile biologically active compounds interacting with cytochromes P450. Chem Biol Interact 139: 1-21, 2002.

12. Manthey JA, Guthrie N and Grohmann K: Biological properties of Citrus flavonoids Pertaining to cancer and inflammation. Curr Med Chem 8: 135-153, 2001.

13. Surh YJ: Cancer chemoprevention with dietary phytochemicals. Nat Rev Cancer 3: 768-780, 2003.

14. Galati G, Teng S, Moridani MY, Chan TS and O Brien PJ: Cancer chemoprevention and apoptosis mechanisms induced by dietary polyphenolics. Drug Metabol Drug Interact 17: 311-349, 2000.

15. Yang CS, Landau JM, Huang MT and Newmark HL: Inhibition of carcinogenesis by dietary polyphenolic compounds. Annu Rev Nutr 21: 381-406, 2001.

16. Banday JA, Shah SA, Kanth AH, Farozi A and Wani H: In Vitro screening for anticancer activity of petroleum ether and ethyl acetate extracts of Conyza canedensis growing in Kashmir region. Adv Biomed Pharma 2: 82-85, 2015.

17. Park GB, Kim DJ, Kim YS, Lee HK, Kim CW and Hur DY: Silencing of galectin-3 represses osteosarcoma cell migration and invasion through inhibition of FAK/Src/Lyn activation and $\beta$-catenin expression and increases susceptibility to chemotherapeutic agents. Int J Oncol 46: 185-194, 2015.

18. Li X, Yang G, Li X, Zhang Y, Yang J, Chang J, Sun X, Zhou X, Guo Y, Xu Y, et al: Traditional Chinese medicine in cancer care: A review of controlled clinical studies published in Chinese. PLoS One 8: e60338, 2013. 